\title{
REFINO DE COMPOSTOS DE LÍTIO GRAU TÉCNICO PARA USOS EM BATERIAS DE ÍON-Li
}

\author{
C. S. PEREIRA ${ }^{1,2}$, C. C. GONÇALVES ${ }^{1}$, G. C. MOREIRA, K. C. B. ALEXANDRE $^{1}$, P. F. A. BRAGA ${ }^{1, *}$ \\ ${ }^{1}$ Centro de Tecnologia Mineral - CETEM, Coordenação de Processamento e Tecnologias Minerais - COPTM, \\ ${ }^{2}$ Universidade Federal do Rio de Janeiro (UFRJ), Escola de Química \\ pbraga@cetem.gov.br*
}

Submetido 16/11/2017 - Aceito 21/11/2017

DOI: $10.15628 /$ holos.2017.6555

\section{RESUMO}

Compostos de lítio com especificações químicas de alto teor de pureza são materiais chave na nova era de sustentabilidade energética; suas aplicações mais importantes visam o armazenamento de grande quantidade de energia, substituindo fontes de energia convencionais (hidrelétrica e combustíveis fósseis) e promovendo a utilização da energia solar e eólica, inclusive durante o período da noite ou de falta de ventos. No Brasil, a indústria de lítio produz tão somente produtos de grau técnico (carbonato e hidróxido de lítio), estando aquém de produtos de lítio grau bateria. É proposto neste trabalho o desenvolvimento de um processo para o refino desses compostos de grau técnico (industrial), de maneira a transformá-los em produto de grau bateria, prevendo sua posterior aplicação na produção de baterias recarregáveis de íonlítio, usadas intensamente como fonte de energia (baterias) em aparelhos e dispositivos com portabilidade, baterias para veículos elétricos e para armazenadores de energia de alta carga (grid storage). 0 processo desenvolvido foi baseado, inicialmente, na caracterização e conhecimento dos contaminantes contidos nos produtos de grau técnico. Conhecidos os principais contaminantes, foi proposta uma rota tecnológica para refino dos compostos de lítio baseados nas suas propriedades químicas e físicas como solubilidade em água. Os resultados obtidos mostram que é possível purificar o carbonato de lítio para atender às especificações para compostos de grau de bateria e, ainda, reduzir significativamente a contaminação do hidróxido de lítio, deixando-o próximo do grau de pureza almejado. O carbonato de lítio refinado continha uma pureza superior a 99,5\%, enquadrando-se em um produto de grau bateria.

PALAVRAS-CHAVE: lítio, carbonato de lítio, baterias de íon-Li, refino, hidróxido de lítio.

\section{REFINING OF LITHIUM COMPOUNDS TECHNICAL DEGREE FOR USE IN Li-ION BATTERIES}

\begin{abstract}
High-purity lithium compounds are key-materials in the new energetic sustainability era; their most important applications aim towards storage of greats amount of energy, replacing energy sources (conventional hydroelectric and fossil fuels) and promoting the use of solar and wind energy, even during the period of the night or absence of winds. A simple purification process for the lithium hydroxide and carbonate compounds is proposed in this paper in an economically viable and sustainable procedure, envisaging subsequent application in the production of rechargeable lithium-ion batteries for portable devices, electric vehicles batteries and energy storage devices in industrial scale. The referred process is based on the knowledge and application of the solubility properties of the contaminants in the involved substances, on a series of
\end{abstract}

consecutive washings and filtrations for the lithium carbonate, and solubilization and precipitation of impurities for the lithium hydroxide, in order to eliminate impurities with low-loss of the material of interest. The obtained results show that for the technical grade lithium carbonate the purification is viable to meet the standards in battery application, while the purity of the technical grade lithium hydroxide was also great improved, nevertheless didn't meet standards for battery application. The lithium carbonate had a $99.5 \%$ of purity following the standards for battery application.

KEYWORDS: lithium; lithium carbonate, purification, Li-ion battery, lithium hydroxide. 


\section{INTRODUÇÃO}

O lítio é o mais leve de todos os metais, tem densidade aproximada da metade da água, além de grande potencial eletroquímico. Devido a estas características, o lítio tornou-se um material estratégico para uma nova geração de baterias recarregáveis (Li-íon) para uso em dispositivos eletrônicos ou em ferramentas elétricas portáteis. Recentemente, o lítio tem sido destacado pelo seu uso na composição de acumuladores de energia com maior potencia específica $(\mathrm{W} / \mathrm{kg}$ ) e energia específica $(\mathrm{Wh} / \mathrm{kg})$, como as baterias de veículos elétricos (EVs), que exigem alta densidade energética e leveza (Rosolem, 2016). Como resultado das suas propriedades intrínsecas, suas aplicações no armazenamento de energia e do fato de não ser considerado um agente poluente (se descartado corretamente e/ou reciclado), o lítio tem sido considerado por muitos como a melhor alternativa aos combustíveis fósseis e é comumente chamado de "petróleo branco" (Jeppson, 1978 e Sttaford, 2016).

Nas últimas décadas, tem sido cada vez mais visível a crescente demanda por lítio no mercado internacional. O lítio ocorre sob várias formas na natureza, principalmente como: cloretos em salmouras continentais, que representam as maiores reservas mundiais (Salar de Atacama, no Chile, Salar del Hombre Muerto, na Argentina, Salar do Uyuni, na Bolívia); na água do mar; em argilas hectoríticas (Nevada, EUA) e em pegmatitos (rochas silicatadas) na forma dos minerais espodumênio (principal fonte de lítio no Brasil, Austrália e Canadá), lepidolita e ambligonita (Goonan, 2016).

Apesar de todo potencial tecnológico e econômico, atualmente, o Brasil produz somente compostos de lítio de qualidade técnica, ou seja, produtos químicos de lítio (carbonato e hidróxido de lítio) com pureza inferior à especificada pelo exigente grupo de fabricantes de baterias recarregáveis (Li-íon), sendo usado principalmente na produção de graxas lubrificantes de alto desempenho, prática comum desde a 1941, e na indústria vitro-cerâmica (Moreno, 2013). O market-share do lítio no mercado internacional é de $39 \%$ no segmento de baterias de Li-íon, enquanto que no mercado nacional é inexistente (França e Braga, 2016).

Face ao contexto acima, é objetivo deste trabalho o desenvolvimento de processo para o refino dos compostos de lítio de grau técnico (industrial), atualmente produzidos pela indústria nacional, de maneira a transformá-los em produto de grau bateria, prevendo sua posterior aplicação na produção de baterias recarregáveis. Este trabalho visa o desenvolvimento de uma alternativa viável e econômica para a purificação de compostos de lítio (carbonato e hidróxido), de forma a aumentar a competitividade da indústria nacional, inserindo o Brasil em um patamar mais elevado da indústria mundial de lítio.

\section{METODOLOGIA}

\subsection{Amostras e reagentes}

Foram utilizadas amostras de carbonato de lítio $\left(\mathrm{Li}_{2} \mathrm{CO}_{3}\right)$, com pureza de $98,50 \%$, e de hidróxido de lítio mono-hidratado $\left(\mathrm{LiOH} . \mathrm{H}_{2} \mathrm{O}\right)$, com pureza de $54,7 \%$, ambas de grau técnico e produzidas nacionalmente. Os principais reagentes utilizados foram o cloreto de bário hidratado $\left(\mathrm{BaCl}_{2} .2 \mathrm{H}_{2} \mathrm{O}\right)$, com pureza de $99,0 \%$, e o hidróxido de cálcio $\left[\left(\mathrm{Ca}(\mathrm{OH})_{2}\right]\right.$, com pureza de $95,0 \%$, ambos fabricados pela VETEC. 


\subsection{Materiais e Equipamentos}

Os ensaios de refino dos compostos de lítio foram realizados em escala de laboratório e envolveram operações de extração, ressolubilização e recristalização do carbonato e do hidróxido de lítio de grau técnico.

Para ambos os processos de refino (carbonato e hidróxido), os materiais e equipamentos utilizados foram: almofariz e pistilo; provetas graduadas diversas; béqueres diversos; béquer de aço inox 2L; kitassato com funil büchner; placa de aquecimento com agitação magnética; agitador mecânico; balança analítica e semi-analítica e medidores de $\mathrm{pH}$.

O controle do processo foi realizado com as técnicas de espectrofotometria de chama (Fotômetro de chama Analyser 910), espectrometria de absorção atômica e analisador de enxofre e carbono (LECO SC 632 Analyser). A análise de Li foi efetuada por espectrometria de absorção atômica, após solubilização da amostra por meio de ataque ácido com $\mathrm{H}_{2} \mathrm{SO}_{4}$ e $\mathrm{HCl}$.

Para efeito de avaliação dos resultados dos ensaios de refino do carbonato e do hidróxido de lítio, o produto nacional e o produto nacional refinado foram comparados, em termos de pureza, com os produtos de grau bateria fornecidos pelos maiores produtores mundiais de lítio: Rockwood e FMC Lithium Division.

\subsection{Purificação do Carbonato de Lítio}

Inicialmente foi realizada uma avaliação da composição química do carbonato de lítio nacional, de forma a identificar possíveis elementos contaminantes. Na Tabela 1 está detalhada a análise química do produto nacional de grau técnico, fornecida pelo fabricante.

Tabela 1. Composição química do carbonato de lítio nacional.

\begin{tabular}{|c|c|c|c|c|c|c|c|c|c|}
\hline $\begin{array}{c}\mathrm{Li}_{2} \mathrm{CO}_{3} \\
\%\end{array}$ & $\begin{array}{c}\mathrm{Cl}^{-} \\
\mathrm{ppm}\end{array}$ & $\begin{array}{c}\mathrm{SO}_{4}^{-2} \\
\%\end{array}$ & $\begin{array}{c}\mathrm{CaO} \\
\% \\
\end{array}$ & $\begin{array}{c}\mathrm{Na}_{2} \mathrm{O} \\
\%\end{array}$ & $\begin{array}{l}\mathrm{K}_{2} \mathrm{O} \\
\mathrm{ppm}\end{array}$ & $\begin{array}{c}\mathrm{Fe}_{2} \mathrm{O}_{3} \\
\mathrm{ppm}\end{array}$ & $\begin{array}{l}\text { R.I.* } \\
\text { ppm }\end{array}$ & $\begin{array}{c}\mathrm{Mg} \\
\mathrm{ppm}\end{array}$ & $\begin{array}{c}\mathrm{H}_{2} \mathrm{O} \\
\% \\
\end{array}$ \\
\hline 98,84 & 10 & 0,38 & 0,03 & 0,15 & 10 & 8 & 10 & 38 & 0,03 \\
\hline
\end{tabular}

Verifica-se que o íon sulfato $\left(\mathrm{SO}_{4}{ }^{-2}\right)$ é um dos principais elementos contaminantes, com uma concentração de $0,38 \%$ no carbonato de lítio. Isso porque, o íon sulfato pode estar associado a íons lítio ou sódio de acordo com a reação de formação da Equação 1, a qual é uma das etapas próprio processo de obtenção do carbonato.

$$
\mathrm{Li}_{2} \mathrm{SO}_{4}+\mathrm{Na}_{2} \mathrm{CO}_{3} \Rightarrow \mathrm{Li}_{2} \mathrm{CO}_{3}+\mathrm{Na}_{2} \mathrm{SO}_{4}
$$

Na Figura 1 estão expressas as solubilidades em água dos reagentes e produtos da Equação 1, propriedade essencial na purificação dos compostos de lítio (Lide, 2015). É notório que sulfato de lítio, carbonato de sódio e sulfato de sódio são produtos com alta solubilidade em água quente; inversamente, o carbonato de lítio tem solubilidade mínima em temperaturas acima de $90^{\circ} \mathrm{C}$. 


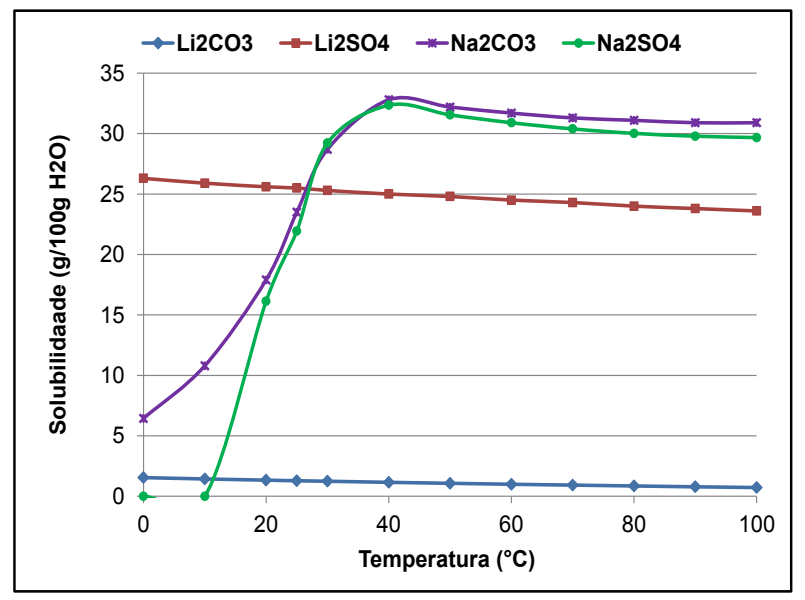

Figura 1 - Curvas de solubilidade dos reagentes e produtos da Eq. 1 em função da temperatura.

Face às diferenças de solubilidade entre o carbonato de lítio (pouco solúvel) e os demais reagentes e produtos da Equação 1, foi proposta uma rota para refino do $\mathrm{Li}_{2} \mathrm{CO}_{3}$, por meio de uma série de extrações em contra corrente do íon sulfato, com água quente e para relações sólido/líquido $\left(\mathrm{Li}_{2} \mathrm{CO}_{3}: \mathrm{H}_{2} \mathrm{O}\right)$ de 1:1 e 1:2. O circuito de extração da rota proposta está apresentado na Figura 2 (Rosales e Braga, 2014).

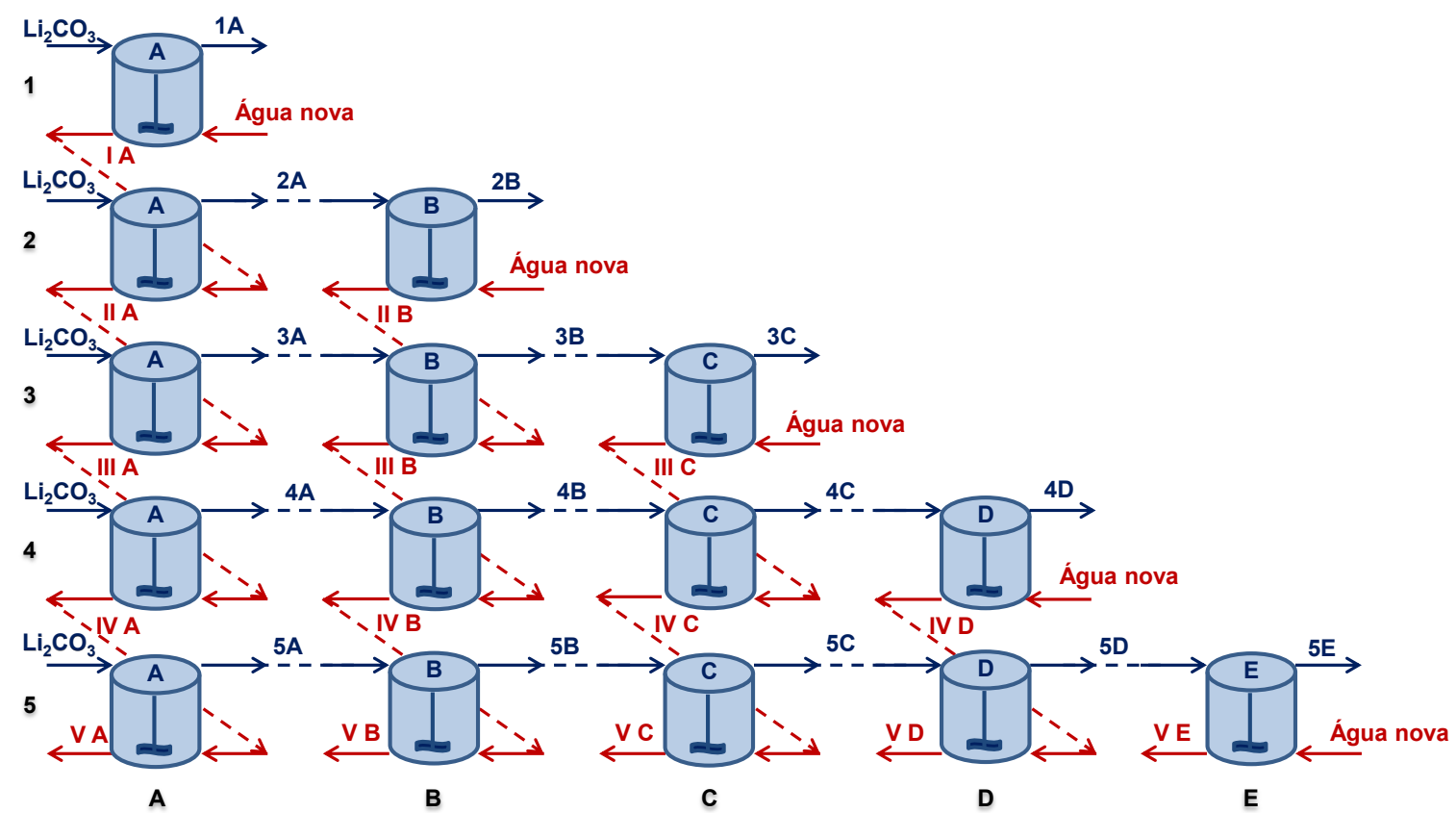

Figura 2. Diagrama do processo de refino do carbonato de lítio.

Cada ensaio foi realizado com 5 etapas de extração aquosa de sulfato (SO4-2). Os produtos finais (carbonatos de lítio) correspondem à numeração em algarismo arábico, enquanto os licores são correspondentes à numeração romana.

\subsection{Purificação do Hidróxido de Lítio}

De maneira análoga à metodologia proposta para purificação do carbonato de lítio, também foi realizada uma avaliação da composição química do hidróxido de lítio nacional, de 
forma a identificar possíveis elementos contaminantes. A análise química do produto nacional de grau técnico fornecido pelo fabricante é detalhada na Tabela 2.

Tabela 2. Composição química do hidróxido de lítio nacional

\begin{tabular}{ccccccccc}
\hline $\begin{array}{c}\mathrm{LiOH} \\
\%\end{array}$ & $\begin{array}{c}\mathrm{CO}_{3}^{-2} \\
\%\end{array}$ & $\begin{array}{c}\mathrm{SO}_{4}^{-2} \\
\%\end{array}$ & $\begin{array}{c}\mathrm{CaO} \\
\%\end{array}$ & $\begin{array}{c}\mathrm{NaOH} \\
\%\end{array}$ & $\begin{array}{c}\mathrm{K}_{2} \mathrm{O} \\
\%\end{array}$ & $\begin{array}{c}\mathrm{Fe}_{2} \mathrm{O}_{3} \\
\text { ppm }\end{array}$ & $\begin{array}{c}\mathrm{R}^{-I} .^{*} \\
\text { ppm }\end{array}$ & $\begin{array}{c}\mathrm{Cl}^{-} \\
\text {ppm }\end{array}$ \\
\hline 54,77 & 0,60 & 0,01 & 0,04 & 0,02 & 0,03 & 3 & 10 & 10 \\
\hline
\end{tabular}

*resíduo insolúvel

Como observado na Tabela 2, o íon carbonato $\left(\mathrm{CO}_{3}^{-2}\right)$ é o principal contaminante com uma concentração de $0,60 \%$ no hidróxido de lítio. Neste caso, o íon carbonato pode estar associado a íons lítio ou cálcio, de acordo com a reação de formação do hidróxido de lítio mostrada na Equação 2.

$$
\mathrm{Li}_{2} \mathrm{CO}_{3}+\mathrm{Ca}(\mathrm{OH})_{2} \Rightarrow \mathrm{LiOH}+\mathrm{CaCO}_{3}
$$

Na Figura 3 verifica-se a solubilidade em água dos reagentes e produtos da Equação 1, sendo destaque que o carbonato de lítio, o hidróxido de cálcio e o carbonato de cálcio são produtos com baixa solubilidade em água quente; de maneira oposta, o hidróxido de lítio tem boa solubilidade em temperaturas acima de $90^{\circ} \mathrm{C}$ (Lide, 2005).

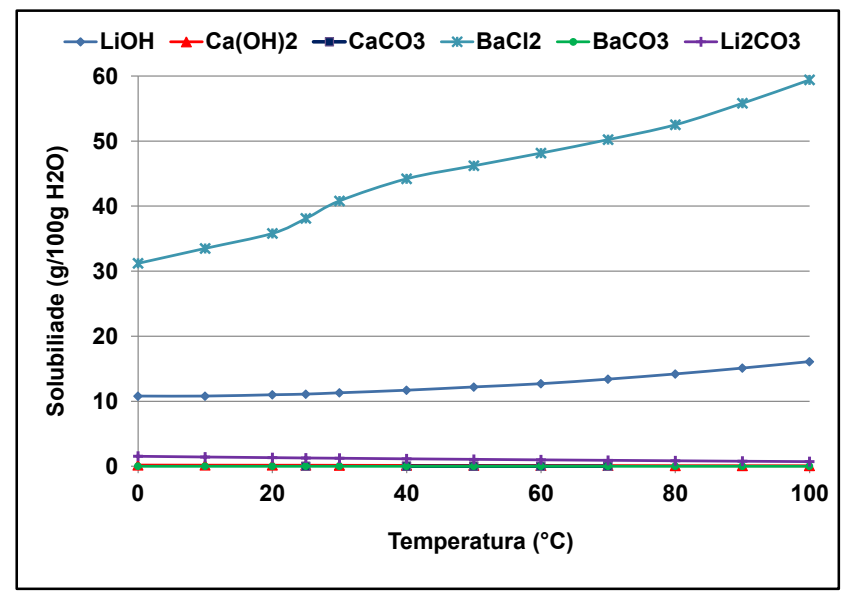

Figura 3. Curvas de solubilidade dos reagentes e produtos da Eq. 2 em função da temperatura

Para eliminação dos íons $\mathrm{CO}_{3}{ }^{-2}$, principal impureza do hidróxido de lítio, foi proposta a precipitação do carbonato contaminante por meio de reação com cloreto de bário $\left(\mathrm{BaCl}_{2}\right)$ ou hidróxido de cálcio, uma vez que os carbonatos resultantes $\left(\mathrm{BaCO}_{3}\right.$ ou $\left.\mathrm{CaCO}_{3}\right)$ são praticamente insolúveis (Equações 3 e 4).

$$
\begin{aligned}
& \mathrm{CO}_{3}^{-2}+\mathrm{BaCl}_{2} \Rightarrow 2 \mathrm{Cl}^{-}+\mathrm{BaCO}_{3} \mathrm{ppt} \\
& \mathrm{CO}_{3}^{-2}+\mathrm{Ca}(\mathrm{OH})_{2} \Rightarrow \mathrm{OH}^{-}+\mathrm{CaCO}_{3} \mathrm{ppt}
\end{aligned}
$$

Para esse efeito, remoção dos carbonatos, foi adicionada uma massa estequiométrica de $\mathrm{BaCl}_{2} \cdot \mathrm{H}_{2} \mathrm{O}$ (solução concentrada) e $\mathrm{Ca}(\mathrm{OH})_{2}$ (suspensão) em uma solução de $\mathrm{LiOH}$, para formação e 
precipitação dos carbonatos insolúveis (Eq. 3 e Eq. 4), que posteriormente serão eliminados por filtração (Figura 4).

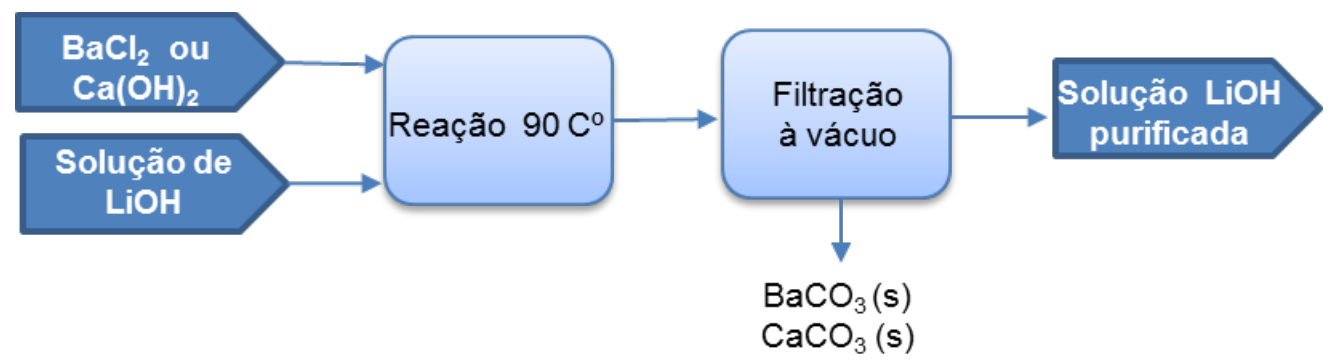

Figura 4. Diagrama simplificado do processo de purificação do hidróxido de lítio.

Esses ensaios de refino foram realizados de modo cinético, isto é, as reações de precipitação dos carbonatos foram contínuas e com aquecimento constante, de modo a aumentar a concentração do licor de hidróxido de lítio, e consequentemente diminuir a solubilidade dos carbonatos. Alíquotas do material reacional foram coletadas e filtradas, sendo o licor filtrado e dosado em termos de carbonato. Não foi atingida a saturação do licor de hidróxido de lítio, de forma a prevenir perdas de lítio.

O licor final de hidróxido de lítio, com concentração próxima ao limite de saturação, foi então resfriado e cristalizado sob forma de $\mathrm{LiOH} . \mathrm{H}_{2} \mathrm{O}$. Os cristais de hidróxido foram filtrados a vácuo, secos sob temperatura controlada e analisados em termos de pureza (\% LiOH).

\section{RESULTADOS E DISCUSSÃO}

\subsection{Refino do Carbonato de Lítio}

Foram realizadas duas sequências de ensaios de refino dos produtos de lítio, com a diferença entre ambas relativa à relação sólido:líquido (carbonato de lítio: água), que foi de 1:1 e $1: 2$.

Verifica-se na Tabela 3 que o carbonato de lítio refinado na relação sólido:líquido de 1:1 atingiu um valor máximo de pureza de 99,32\%, após 5 etapas de extração. Esse valor de pureza é superior a do produto nacional, porém inferior às dos produtos internacionais. Já no ensaio com relação sólido:líquido de $1: 2$, atingiu-se $99,74 \%$ de pureza, com apenas uma etapa de extração; esse resultado é superior ao valor mínimo internacional de $99,5 \%$, para o produto equivalente grau bateria.

Tabela 3. Processo de purificação de $\mathrm{Li}_{2} \mathrm{CO}_{3}$ (relação sólido:líquido 1:1 e 1:2).

\begin{tabular}{|c|c|c|c|c|c|c|c|c|}
\hline & \multirow{2}{*}{$\begin{array}{c}\text { Nacional grau } \\
\text { técnico }\end{array}$} & \multicolumn{5}{|c|}{ Purificado CETEM } & \multirow{2}{*}{$\begin{array}{c}\text { FMC } \\
\text { grau bateria }\end{array}$} & \multirow{2}{*}{$\begin{array}{l}\text { Rockwood } \\
\text { grau bateria }\end{array}$} \\
\hline & & Etapa 1 & Etapa 2 & Etapa 3 & Etapa 4 & Etapa 5 & & \\
\hline \multicolumn{9}{|c|}{ relação sólido/líquido (1:1) } \\
\hline $\begin{array}{c}\mathrm{Li}_{2} \mathrm{CO}_{3} \\
\% \\
\end{array}$ & 98,84 & 96,98 & 98,68 & 98,4 & 99,02 & 99,32 & 99,5 (mín.) & 99,8 (mín.) \\
\hline \multicolumn{9}{|c|}{ relação sólido/líquido (1:2) } \\
\hline $\begin{array}{c}\mathrm{Li}_{2} \mathrm{CO}_{3} \\
\% \\
\end{array}$ & 98,84 & & 99,76 & 99,76 & 99,76 & 99,77 & 99,5 (mín.) & 99,8 (mín.) \\
\hline
\end{tabular}




\subsection{Refino do hidróxido de lítio}

$\mathrm{Na}$ Figura 4A tem-se a curva de extração de carbonatos em função da concentração de hidróxido de lítio, após o tratamento com cloreto de bário. Na concentração de $40 \mathrm{~g} / \mathrm{L}$ de hidróxido de lítio, o teor residual de $\mathrm{CO}_{3}{ }^{2-}$ foi de $0,18 \%$.
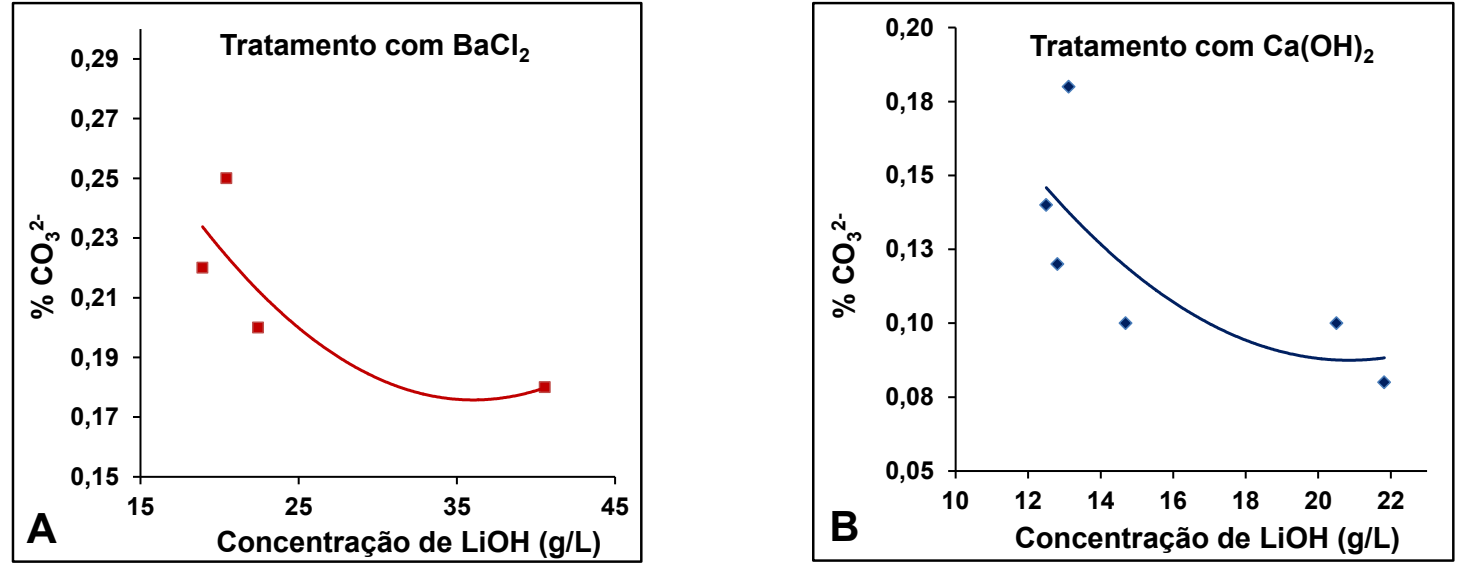

Figura 4. Curvas de extração de carbonato presente no hidróxido de lítio nacional.

Na Figura 4B está contida a curva de extração de carbonatos em função da concentração de hidróxido de lítio, após o tratamento com hidróxido de cálcio. Na concentração de $22 \mathrm{~g} / \mathrm{L}$ de hidróxido de lítio, o teor residual de CO32- era de 0,08\%. É claro, portanto, a maior eficiência de remoção dos íons carbonato (CO32-) no tratamento com hidróxido de cálcio.

O licor final de hidróxido de lítio submetido ao tratamento com hidróxido de cálcio foi concentrado até valores próximos da saturação, resfriado e cristalizado sob forma de LiOH.H2O. O hidróxido de lítio refinado com hidróxido de cálcio continha uma pureza de 55,15\%, maior que a pureza do produto nacional, porém ainda inferior aos requisitos mínimos para um produto grau bateria, o qual é $56,5 \%$ (Tabela 4 ).

Tabela 4. Purificação do hidróxido de lítio após tratamento com hidróxido de cálcio.

\begin{tabular}{c|c|c|c|c}
\hline LiOH. $\mathbf{H}_{2} \mathrm{O}$ & $\begin{array}{c}\text { grau técnico } \\
\text { nacional }\end{array}$ & $\begin{array}{c}\text { purificado } \\
\text { com cálcio }\end{array}$ & $\begin{array}{c}\text { FMC } \\
\text { grau bateria }\end{array}$ & $\begin{array}{c}\text { Rockwood } \\
\text { grau bateria }\end{array}$ \\
\hline Pureza & $54,77 \%$ & $55,15 \%$ & $56,50 \%$ & $56,50 \%$ \\
\hline
\end{tabular}

\section{CONCLUSÕES}

Após os ensaios realizados, constatou-se a possibilidade de purificação do carbonato de lítio $\left(\mathrm{Li}_{2} \mathrm{CO}_{3}\right)$ nacional de grau técnico, cuja pureza era de $98,5 \%$, transformando-o em um produto grau bateria. No processo de refino estudado, tem-se que o carbonato de lítio refinado com apenas uma etapa de extração de sulfato $\left(\mathrm{SO}_{4}{ }^{2-}\right)$, principal contaminante, e com uma relação sólido:líquido de 1:2, atingiu uma pureza de $99,74 \%$.

No caso do hidróxido de lítio, o produto refinado com hidróxido de cálcio apresentou uma pureza de 55,15\%, maior que a pureza do produto nacional, porém ainda inferior aos requisitos mínimo para um produto grau bateria, o qual é comercializado internacionalmente $(56,5 \%$ de pureza). 


\section{REFERÊNCIAS}

Braga, P., \& França, S. (2013). Série Estudos e Documentos 81 - Lítio: Um Mineral Estratégico. Rio de Janeiro: CETEM/MCTI.

França, S., \& Braga, P. (2016). Aplicações dos compostos de lítio: mercados internacional, nacional e perspectivas. II Seminário Sobre Lítio-Brasil.

Goonan, T. (2016). Lithium Use in Batteries.

Jeppson, D., Ballif, J., Yuan, W., \& Chou, B. (1978). Lithium literature review: Lithium's properties and interactions.

Lide, D. (2005). Handbook of chemistry and physics (85th ed., pp. Press. Chapter 4, p. 49-86. Chapter 8, p. 123-128.).

Moreno, L. (2013). A strategic energy metal. Significant increase in demand ahead. Euro Pacific Canada.

Rosales, G. (2014). Estudio de la extracción de Li a partir de $\alpha$-espodumeno por lós métodos ácido y alcalino (1st ed.).

Rosolem, M. (2016). Bateria de Lítio-íon-Estado da Arte. Il Seminário Sobre Lítio-Brasil.

Stafford, J. (2017). Lithium war heats up after launch of Tesla Model 3. Oilprice.com. http://www.mineweb.com/news/energy/lithium-war-heats-up-after-launch-of-tesla-model3/. Acessado em 28 de Fevereiro de 2017. 